\title{
Signal Denoising Method Based on Improved Wavelet Threshold Function for Microchip Electrophoresis $C^{4} D$ Equipment
}

\author{
Yaonan Tong $(D$, Jingui Li, Yaohui Xu, and Lichen Cao \\ School of Information Science and Engineering, Hunan Institute of Science and Technology, Yueyang, Hunan 414006, China \\ Correspondence should be addressed to Yaonan Tong; tongyaon@hnist.edu.cn
}

Received 24 April 2020; Accepted 19 June 2020; Published 10 July 2020

Guest Editor: Shao-Bo He

Copyright (c) 2020 Yaonan Tong et al. This is an open access article distributed under the Creative Commons Attribution License, which permits unrestricted use, distribution, and reproduction in any medium, provided the original work is properly cited.

\begin{abstract}
A signal denoising method using improved wavelet threshold function is presented for microchip electrophoresis based on capacitively coupled contactless conductivity detection $\left(M E-C^{4} D\right)$ device. The evaluation results of denoising effect for the ME$\mathrm{C}^{4} \mathrm{D}$ simulation signal show that using Daubechies 5 (db5) wavelet at a decomposition level 4 can produce the best performance. Furthermore, the denoising effect is compared with, as well as proved to be superior to, the existing techniques, such as Savitzky-Golay, Fast Fourier Transform, and soft threshold method. This method has been successfully applied to the selfdeveloped $\mathrm{ME}-\mathrm{C}^{4} \mathrm{D}$ equipment. After executing this method, the noise is cleanly removed, and the signal peak shape and peak area are well maintained.
\end{abstract}

\section{Introduction}

Microfluidic technology, especially microchip electrophoresis based on capacitively coupled contactless conductivity detection $\left(\mathrm{ME}-\mathrm{C}^{4} \mathrm{D}\right)[1-3]$, has become a very important and promising branch of miniaturized total chemical analysis systems ( $\mu$-TAS) [4-6]. Because of its advantages of little samples and reagents consumption, fast analysis speed, high separation efficiency, and convenient miniaturization, microfluidic technology has been widely used in different fields, such as biomedicine [7, 8], food inspection [9], environmental monitoring [10], clinical application [11], and so on.

The ME- $C^{4} \mathrm{D}$ device analyzes the ion composition in solution by detecting the change of electrical conductivity based on the coupling capacitance between the electrode and insulation layer of the chip [12-15]. Therefore, it can effectively avoid some troubles of electrochemical contact detect method, such as electrode scaling, electrolysis bubble, electric field interference, and so on [13-16]. But the ME$\mathrm{C}^{4} \mathrm{D}$ device has poor anti-electromagnetic interference ability and low sensitivity [17-19]. The inherent noise of the system and the structure of the microfluidic chip will cause the ME- $C^{4} \mathrm{D}$ signal to be disturbed, thus affecting the analysis of the detection results and reducing its accuracy. Therefore, finding an appropriate method for signal denoising is an extremely important processing step before analysis and diagnosis.

The traditional signal denoising methods mainly include Fourier transform and curve fitting method. The Fourier transform is simple and easy to implement, but it is difficult to solve the noise filtering of nonstationary signals. The curve fitting method has high accuracy, but there are difficulties in selecting fitting points. The wavelet transform (WT), that developed rapidly from the 1980s, can fully highlight the characteristics of some aspects of problem, which has been widely used in capillary electrophoresis (CE) signal denoising $[20,21]$. Furthermore, the wavelet threshold denoising developed from WT has better performance [22]. The denoising effect of this method mainly depends on the selection of threshold function. Some traditional threshold functions such as hard threshold and soft threshold are widely used for signal denoising due to the simple structure and good efficiency. For example, Liu uses hard threshold function to denoise microchip CE signal sampled from a home-built laser-induced fluorescence detection system [19]. Zhang et al. improve the soft threshold function to enhance the electrochemiluminescence CE signal denoising 
effect [22]. The above WT methods can achieve excellent denoising effect, but paid less attention to the maintenance of wave peak area, which is a very important feature for analyzing the ME- ${ }^{4} \mathrm{D}$ signal, and can reflect the content of the ion component to be detected [23]. Therefore, they are not very suitable for the ME- $\mathrm{C}^{4} \mathrm{D}$ signal.

The objective of this work is to develop an effective denoising method with minimal impact on the signal peak shape and area for improving the performance of the ME$C^{4} \mathrm{D}$ device. We have improved the wavelet threshold function according to the ME- $\mathrm{C}^{4} \mathrm{D}$ signal characteristics. Evaluation of denoising effect for the ME- ${ }^{4} \mathrm{D}$ simulation signal has been done by selecting various wavelets and different decomposition levels, and it was found that db5 wavelet at level 4 is the optimal solution. Furthermore, the proposed method has been successfully applied to the selfdeveloped ME- $\mathrm{C}^{4} \mathrm{D}$ equipment for signal denoising.

\section{Materials and Methods}

2.1. Chemicals. Potassium chloride, sodium chloride, lithium chloride sample solution, and MES-His buffer solution were of analytical grade, purchased from Sinopharm Chemical Reagent Co., Ltd. All chemicals were degassed ultrasonically for 5 minutes before being used and filtered with a $0.22 \mu \mathrm{m}$ pore water microfiltration membrane.

2.2. Apparatus. A self-developed $M E-C^{4} \mathrm{D}$ equipment used in the experiment described is shown in Figure 1. The microchip (cross-shaped structure; the chip separation channel length is $50 \mathrm{~mm}, 50 \mu \mathrm{m}$ in width, and $25 \mu \mathrm{m}$ in depth) was placed on the testing table of the detector. Under a given high-voltage electric field, the directional migration of the microchannel solution will occur. When the ions flow through the detector at the end of the channel, the induced current signal will be converted to a voltage signal and finally displayed on the computer after a series of processing steps. Among them, the control of voltage, sample injection time, and signal waveform can be controlled by the computer. The monitoring software on the PC terminal was self-developed.

2.3. Noise Sources. The analysis channel in the microfluidic chip is micron level, and the noncontact conductance detection is completed based on capacitive coupling principle. Therefore, the detected ME- $C^{4} D$ signal is very weak and will be disturbed by a lot of noise. In order to eliminate noise effectively, it is necessary to analyze the cause of noise and take corresponding measures to deal with it according to different noise sources.

According to the ME- $\mathrm{C}^{4} \mathrm{D}$ signal detection principle and experimental results, the interference and noise of the solution electrical conductivity signal mainly come from two sources. One is the inherent noise of the system, such as the external noise signal from the detection circuit caused by the action of electricity, magnetism, etc., which can be directly filtered through the hardware in the microfluidic chip detection device. The other is high-frequency noise, which is caused by the fast acquisition rate, microchip, detect circuits,

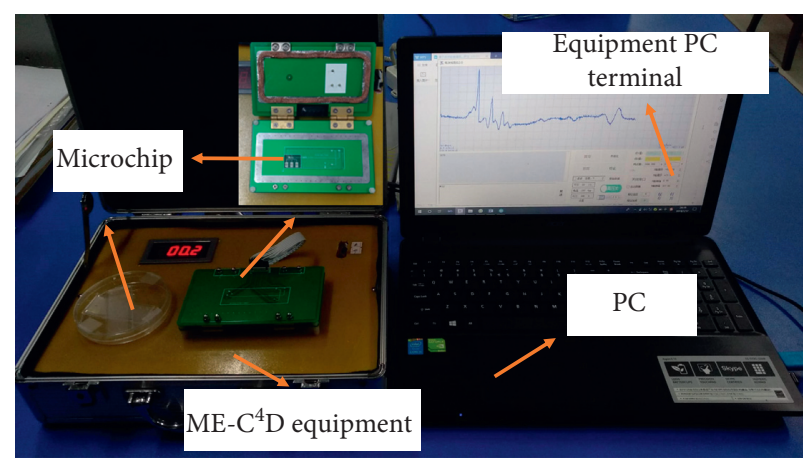

Figure 1: Self-developed ME- $C^{4} \mathrm{D}$ equipment.

environmental disturbances, and so on [24]. It is difficult to filter by optimizing the hardware design of the $\mathrm{ME}-\mathrm{C}^{4} \mathrm{D}$ equipment. The threshold method based on wavelet transform has a good denoising performance. Therefore, this paper studies the appropriate denoising method based on wavelet transform.

2.4. Wavelet Thresholding Denoising Theory. It is assumed that the ME-C $\mathrm{C}^{4} \mathrm{D}$ signal with noise which is collected by microchip electrophoresis analysis device can be expressed as

$$
f(i)=s(i)+n(i), \quad i=0,1,2, \ldots, N-1,
$$

where $f(i)$ is the ME-C $C^{4} \mathrm{D}$ signal with noise, $s(i)$ is a pure ME- $C^{4} \mathrm{D}$ signal, and the noise signal is represented by $n(i)$. Because it is very difficult to recover $s(i)$ directly from the noisy signal $f(i)$, the useful signal and noise can be separated by processing the corresponding wavelet decomposition coefficients according to the different characteristics of it's in the wavelet transform. In practical applications, the useful signals are usually in the low frequency band, while the noise signals are usually in the high-frequency band. According to this characteristic, we can firstly decompose the signal by wavelet transform. As an example, three-level decomposition is shown in Figure 2. The noise-containing signal is decomposed into a low-frequency coefficient (CA1) and a high-frequency coefficient (CD1). CA1 can be further decomposed to form a new low-frequency coefficient (CA2) and a new high-frequency coefficient (CD2). With the increase of the decomposition level, the wavelet coefficient amplitude of the useful signal is basically unchanged, while the amplitude of wavelet coefficient of noise is rapidly attenuated to zero [25]. Therefore, the wavelet coefficients after decomposition can be processed by selecting appropriate threshold function, and then the signal can be reconstructed to achieve the effect of denoising.

2.5. Denoising by Improved Threshold Function. The wavelet coefficients of the ME- $\mathrm{C}^{4} \mathrm{D}$ signal have strong correlation at different decomposition levels, while the wavelet coefficients of the noise are weak or irrelevant [26], which is suitable for the denoising range of wavelet transform. The ME- ${ }^{4} \mathrm{D}$ 


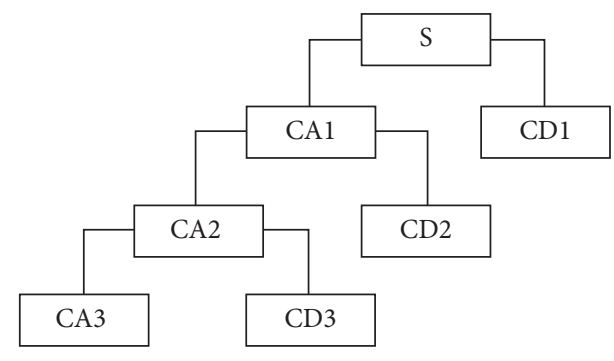

Figure 2: Signal three-level wavelet decomposition diagram.

signal denoising procedure by wavelet transform is as follows:

(1) A suitable wavelet basis function similar to the original ME- $\mathrm{C}^{4} \mathrm{D}$ signal is selected and the number of decomposition layers is determined. The $J$ layer wavelet decomposition of the noise $\mathrm{ME}-\mathrm{C}^{4} \mathrm{D}$ signal is carried out by using Mallet algorithm, and the high-frequency coefficient components and lowfrequency coefficient components of different decomposition scales are obtained.

(2) Select the threshold and use a threshold function to quantify the high-frequency wavelet coefficients from layer 1 to layer $J$.

(3) The low-frequency coefficients of layer $J$ and the high-frequency coefficients of layer 1 to layer $J$ processed by threshold function are inversely transformed to obtain the denoised $\mathrm{ME}-\mathrm{C}^{4} \mathrm{D}$ signal.

The denoising effect mainly depends on the selection threshold and the design of threshold function in step (2). In this work, the classical fixed threshold is adopted, and its expression is

$$
\lambda=\sigma \sqrt{2 \lg N}
$$

where $\lambda$ is the threshold, $N$ is the length of the $M E-C^{4} D$ signal, and $\sigma$ represents the standard deviation of the noise signal. $\sigma$ is used to measure the strength of the noise signal, and $\sigma=\operatorname{median}\left(w_{j k}\right) / 0.6745$, where median $(\cdot)$ means the return median function.

There are two classical types of threshold functions for wavelet denoising, that is, hard and soft threshold. The soft threshold function is more representative. It can be defined as

$$
\widehat{w}_{j k}= \begin{cases}w_{j k}-\lambda, & w_{j k} \geq \lambda, \\ 0, & \left|w_{j k}\right|<\lambda, \\ w_{j k}+\lambda, & w_{j k} \leq-\lambda,\end{cases}
$$

where $w_{j k}$ is the wavelet coefficient of the original signal after wavelet transform, $\hat{w}_{j k}$ is the estimated wavelet coefficient after quantification, and $\lambda$ is the threshold. The soft threshold function graph is depicted in Figure 3.

It can be seen from Figure 3 that the soft threshold function has a good continuity, which overcomes the shortcomings of discontinuity in the hard threshold

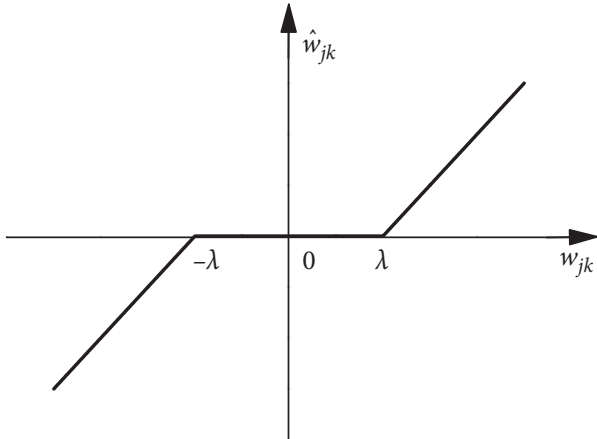

FIgURE 3: Soft threshold function graph.

function and solves the problem that some oscillations will occur in the reconstructed signal. However, there is a constant deviation between original and estimated value of the wavelet coefficient. It is bound to make the reconstructed signal produce distortion and reduce the accuracy. In addition, the wavelet denoising principle illustrates that the smaller wavelet coefficients are also composed of useful signals and noises [27]. However, according to equation (3), if the wavelet coefficients with absolute values smaller than the threshold are set to zero, some useful signal information will be lost and thus the signal-to-noise ratio (SNR) will be reduced.

In order to overcome the above defects of soft threshold function and improve the denoising effect, a new threshold function should meet the following requirements:

(1) The function is continuous at the threshold points.

(2) The constant deviation between the original and quantized wavelet coefficients is reduced as much as possible. However, if the deviation is reduced to zero, it will become a hard threshold function, which cannot achieve the improvement effect [25].

(3) Retain some useful signal information in smaller wavelet coefficients to reduce signal distortion.

Based on the three above requirements, this paper introduces nonlinear function and variable parameters and proposes an improved threshold function:

$$
\widehat{w}_{j k}= \begin{cases}w_{j k}+\operatorname{sign}\left(w_{j k}\right) \cdot\left(-\lambda+a \lambda^{3}\right), & \left|w_{j k}\right| \geq \lambda, \\ a w_{j k}^{3}, & \left|w_{j k}\right|<\lambda,\end{cases}
$$

where $a$ is a variable parameter less than $1 / \lambda^{2}$. The improved threshold function has continuity and overcome the defect of constant deviation of soft threshold function. By multiplying $a$ with the smaller wavelet coefficient $w_{j k}$, the removal ratio of noise signal can be controlled by adjusting the value of $a$. By this mean, the useful signal information in the small wavelet coefficients can be retained flexibly, so the denoised signal is closer to the original one. These properties of this improved threshold function and the corresponding proof are presented as follows:

(1) Continuity analysis is 


$$
\begin{gathered}
\lim _{w_{j k} \longrightarrow-\lambda^{-}} \widehat{w}_{j k}=\lim _{w_{j k} \longrightarrow-\lambda^{+}} \widehat{w}_{j k}=-a \lambda^{3}, \\
\lim _{j k} \widehat{w}_{j k}=\lim _{w_{j k} \longrightarrow \lambda^{+}} \widehat{w}_{j k}=a \lambda^{3} .
\end{gathered}
$$

The threshold function is continuous at threshold $\lambda$ and $-\lambda$. Therefore, the threshold function is continuous over the whole real number field.

(2) Progressive analysis is

$$
\begin{array}{r}
\lim _{w_{j k} \longrightarrow+\infty} \frac{\widehat{w}_{j k}}{w_{j k}}=\lim _{w_{j k} \longrightarrow+\infty}\left(1+\frac{a \lambda^{3}-\lambda}{w_{j k}}\right)=1, \\
\lim _{w_{j k} \longrightarrow-\infty} \frac{\widehat{w}_{j k}}{w_{j k}}=\lim _{w_{j k} \longrightarrow-\infty}\left(1+\frac{\lambda-a \lambda^{3}}{w_{j k}}\right)=1 .
\end{array}
$$

When $w_{j k} \longrightarrow \infty, \widehat{w}_{j k}$ takes $\widehat{w}_{j k}=w_{j k}$ as the asymptote and approaches $w_{j k}$ infinitely, so as to reduce the deviation between the original and the threshold quantized wavelet coefficients as much as possible.

The comparison between hard, soft, and improved function is shown in Figure 4. When $a$ is 0 , the improved threshold function is equivalent to the traditional soft threshold function, and when $a \longrightarrow 1 / \lambda^{2}$, the estimated value $\hat{w}_{j k}$ is closer to the original wavelet coefficient $w_{j k}$. The parameters can be adjusted freely to denoising different signals. Therefore, the improved threshold function was significantly better than the soft threshold function at adaptive ability and flexibility.

\section{Results and Discussion}

3.1. Denoising of the $M E-C^{4} D$ Simulation Signal. The ME$\mathrm{C}^{4} \mathrm{D}$ signal has some peaks, generally represented as Gaussian peaks, reflecting the characteristic spectral lines of a particular substance. When analyzing the substance composition, the obtained peaks' spectra are compared with the characteristic spectral line of some substances. Then, the detected peaks' spectra can indicate which components are contained in the mixture [28].

Due to the lack of standard testing equipment, the simulation means are often used in the research of $\mu$-TAS signal denoising $[22,29]$. When the effectiveness is verified by simulation, the method is applied to the self-developed instrument. Based on the simulation model to capillary electrophoresis signal [29], and according to the characteristics of actual ME- $\mathrm{C}^{4} \mathrm{D}$ signal, a mathematical model of simulation signal was established as follows:

$$
\begin{aligned}
I= & \frac{A_{1}}{\sqrt{2 \pi \sigma_{1}}} e^{-\left(\left(t-t_{1}\right)^{2} / 2 \sigma_{1}^{2}\right)}+\frac{A_{2}}{\sqrt{2 \pi \sigma_{2}}} e^{-\left(\left(t-t_{2}\right)^{2} / 2 \sigma_{2}^{2}\right)} \\
& +\frac{A_{R}}{\sqrt{2 \pi \sigma_{R}}} e^{-\left(\left(t-t_{R}\right)^{2} / 2 \sigma_{R}^{2}\right)}+I_{0},
\end{aligned}
$$

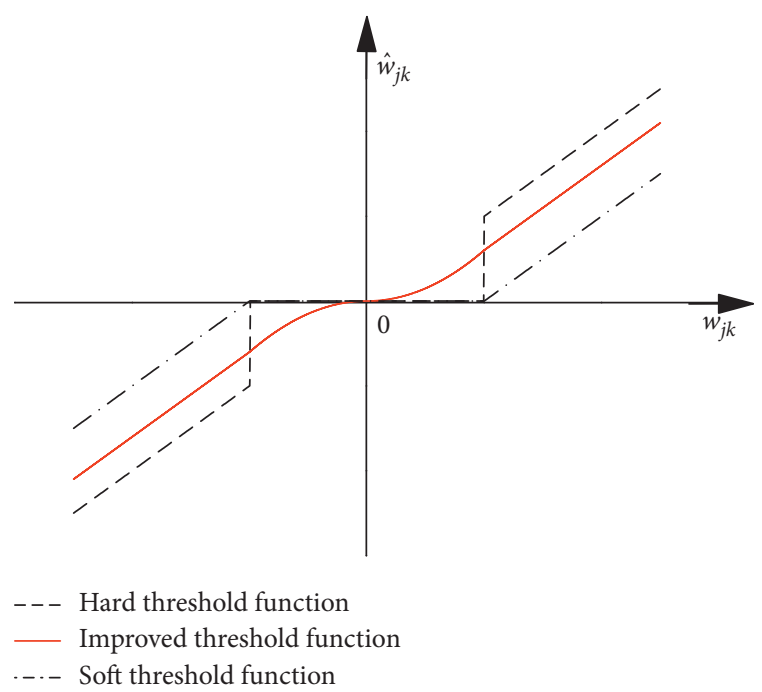

FIGURE 4: Improved threshold function graph.

where $I$ is the simulation ME-C ${ }^{4} \mathrm{D}$ signal, $A_{R}$ is the area of the peak, $t_{R}$ represents the central peak position, $\sigma_{R}$ represents the half peak width, and $I_{0}$ is used to adjust the position of the baseline.

As the Gaussian white noise is close to the noise in the actual ME- $\mathrm{C}^{4} \mathrm{D}$ signal, a certain proportion of Gaussian white noise was added to an ideal signal. The mathematical model can be expressed as follows:

$$
f=I+\beta \cdot \text { noise, }
$$

where noise is Gaussian white noise signal, and $\beta$ is the proportional coefficient. The ideal and noisy signals are simulated and shown in Figure 5.

3.1.1. Evaluation of Denoising Effect. In order to intuitively compare the denoising effect of different methods on ME$\mathrm{C}^{4} \mathrm{D}$ simulation signals, the following two evaluation indexes were introduced:

(1) Signal-to-noise ratio (SNR) is

$$
\mathrm{SNR}=10 \log \frac{\sum_{i=1}^{N} f^{2}(i)}{\sum_{i=1}^{N}[f(i)-\widehat{f}(i)]^{2}}(\mathrm{~dB}) .
$$

(2) Root mean square error (RMSE) is

$$
\mathrm{RMSE}=\sqrt{\frac{1}{n} \sum_{i=1}^{N}[f(i)-\widehat{f}(i)]^{2}}
$$

where $f(i)$ is the original signal, $\hat{f}(i)$ is the reconstructed signal after denoising, $n$ is the sampling points, and $N$ is the signal length. The above two equations indicate that the larger SNR is, the smaller RMSE is, and the better signal denoising effect is. 


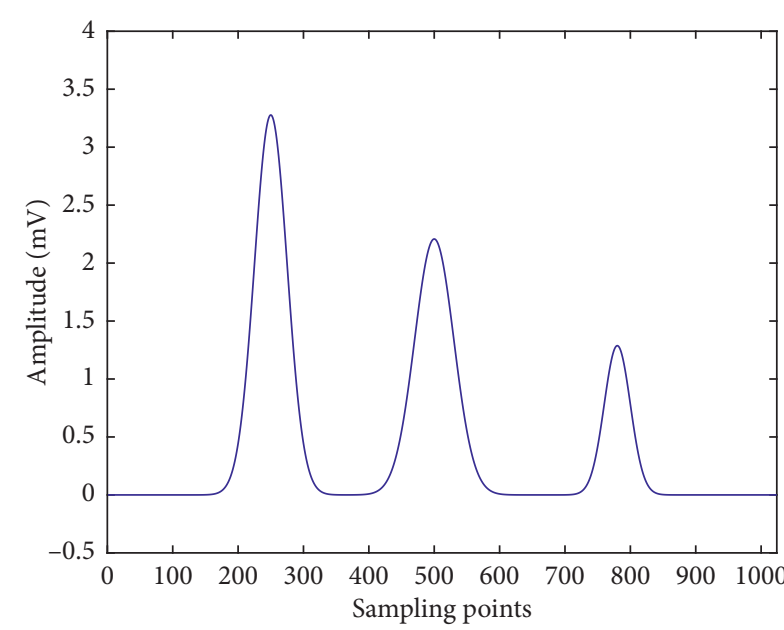

(a)

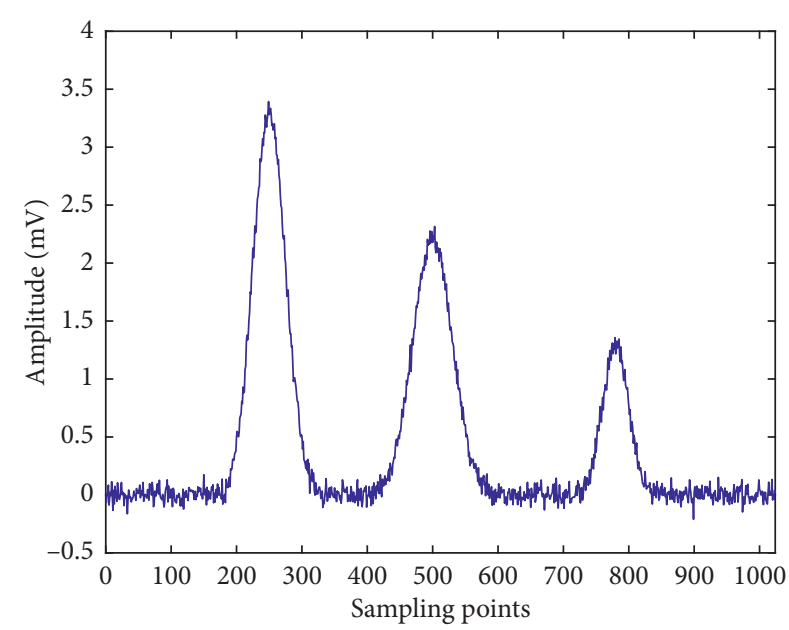

(b)

Figure 5: Simulation ME-C $\mathrm{C}^{4}$ signal and its noisy signal. (a) Simulation ideal signal. (b) Simulation noisy signal.

3.1.2. Selection of Wavelet Basis and Decomposition Level. Using different wavelet basis functions to denoise the same type of signal will produce different effects. Generally, wavelet bases should have the following characteristics: linear phase, short support, higher vanishing distance, etc. [30]. However, few wavelet bases can have these characteristics at the same time. In this paper, several common wavelet bases were selected for denoising experiments. The comparative analysis of common wavelet bases characteristics is shown in Table 1.

In order to select the optimal wavelet basis and obtain the best denoising effect, three different wavelet bases of $\mathrm{db} 1 \sim \mathrm{db} 9$, sym1 sym 9 , and coif1 coif5 were evaluated in this paper, and the improved threshold function was used to denoise the signal containing noise. The SNR and RMSE curves of the denoised signals are shown in Figure 6.

After denoising with db5 wavelet basis, the SNR is the largest, and the RMSE is the smallest, so the denoising effect is the best (Figure 6). Therefore, db5 wavelet basis was used in the following experiments.

In the process of signal denoising by wavelet transform, the selection of appropriate decomposition layers is also very important problem. On the one hand, the larger the number of decomposition layers, the greater the difference between noise and signal performance, and the easier it is to separate. On the other hand, too many layers will make the reconstructed signal more distorted, which will affect the denoising effect to a certain extent. Therefore, it is necessary to deal with this contradiction strictly when selecting the level of decomposition for getting better denoising effect.

On the premise of selecting $\mathrm{db} 5$ wavelet basis, the denoising effect of simulated ME- $\mathrm{C}^{4} \mathrm{D}$ signal under different decomposition layers is depicted in Figure 7. It shows that the 4-layer decomposition can get a good signal denoising effect.

3.1.3. Comparison with Other Denoising Methods. In order to verify the denoising effect of the proposed method, different methods were used to carry out the simulation experiment for the ME- $C^{4} \mathrm{D}$ signal with different SNR Gaussian white noise. The SNR and RMSE comparison data for the denoise signal are shown in Table 2.

The denoising method presented in this paper has the highest SNR and the lowest RMSE; the denoising effect was significantly better than other methods (Table 2 , shown in boldface).

Figure 8 shows the denoising results by the Savitzky-Golay, Fast Fourier Transform, soft threshold method, and the proposed method, respectively. By analyzing the waveform after denoising, it can be seen that the Savitzky-Golay and Fast Fourier Transform were used to denoise the signal, the noise is still apparent (Figures 8(a) and 8(b)). The ME- $C^{4} \mathrm{D}$ signal after denoising by the soft threshold method the signal baseline is smooth, but part of the characteristic signal information is lost (Figure 8(c)). After denoising with the proposed method, the noise is basically removed, the baseline is smooth, the characteristic signal is well retained, and the reconstructed signal after denoising is closer to the original signal (Figure $8(\mathrm{~d})$ ). Therefore, the proposed method has more advantages over the above methods for ME- $C^{4} \mathrm{D}$ signal denoising.

3.2. Application. After theoretical analysis and simulation verification, the proposed method was applied to denoising for the actual signal, which was detected by self-developed ME-C4D and delivered to PC terminal.

The buffer solution of $10 \mathrm{mM}$ MES-His ( $\mathrm{pH}$ 6.15) and the target sample solution of $0.1 \mathrm{mM}$ were introduced to the corresponding position of the microchip and then placed on the detection table of the self-developed equipment. Set the excitation signal source parameter $f=200 \mathrm{kHz}, \mathrm{Vpp}=60 \mathrm{~V}$, high voltage injection $1 \mathrm{~s}$; the collected ME- $\mathrm{C}^{4} \mathrm{D}$ signals are shown in Figure 9.

The transverse axis is the number of sampling points; every 10 sampling points represent the time of $1 \mathrm{~s}$. The longitudinal coordinate represents the amplitude, and the 
TABLE 1: Common wavelet bases characteristics.

\begin{tabular}{lccc}
\hline Basis & $\mathrm{dbN}$ & symN & coif $N$ \\
\hline Symmetry & Approximate symmetry & Approximate symmetry & Approximate symmetry \\
Orthogonality & Have & Have & Have \\
Compact support & Have & Have & Have \\
Support length & $2 N-1$ & $2 N-1$ & $6 N-1$ \\
Filter length & $2 N$ & $2 N$ & $6 N$ \\
Vanishing moments & $N$ & $N$ & $2 N$ \\
\hline
\end{tabular}

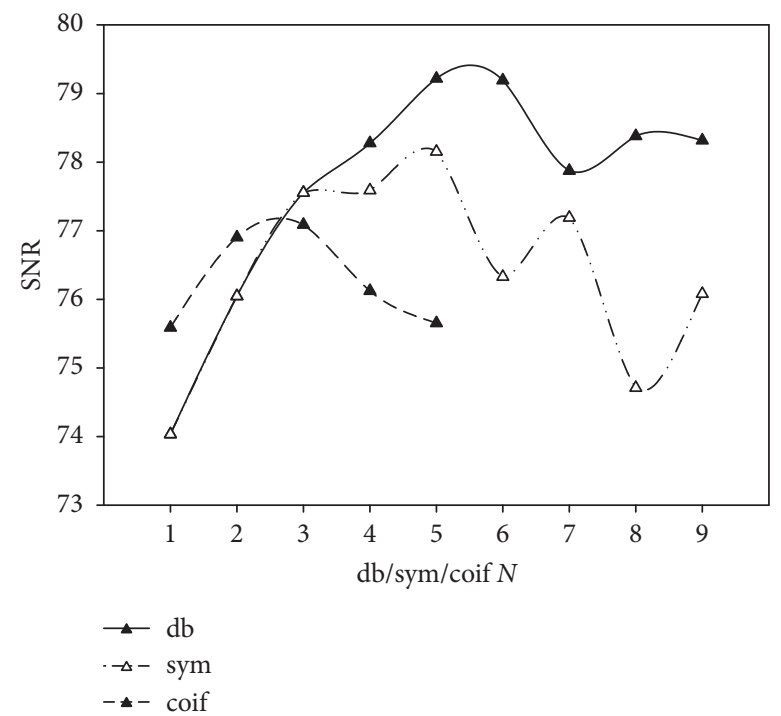

(a)

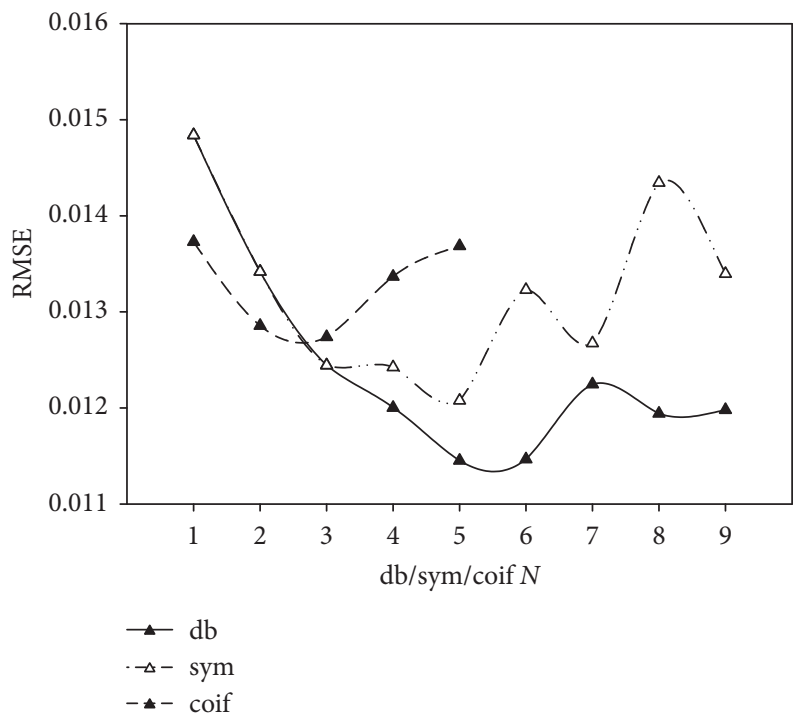

(b)

FIGURE 6: SNR and RMSE after denoising with different wavelet bases. (a) SNR after denoising with different wavelet bases. (b) RMSE after denoising with different wavelet bases.

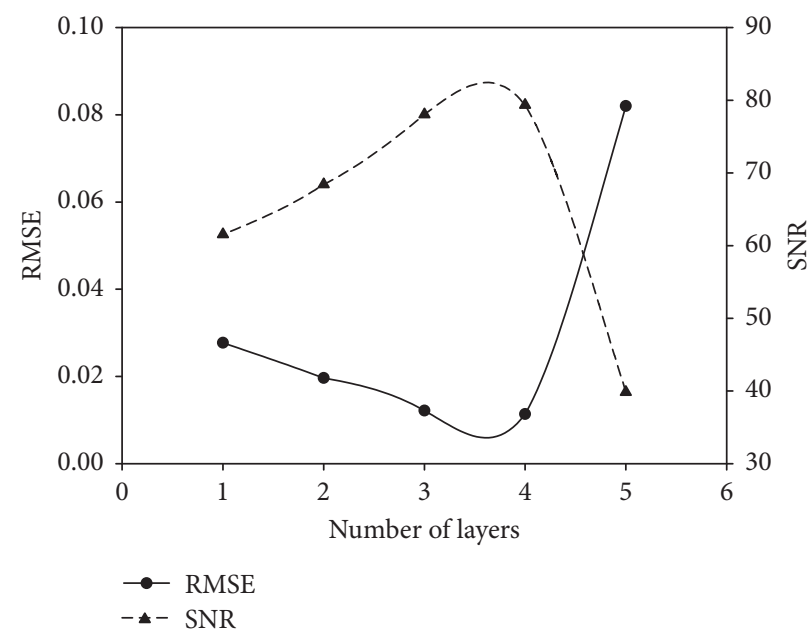

FIGURE 7: SNR and RMSE after denoising at different decomposition levels.

three wave peaks represent $\mathrm{K}^{+}, \mathrm{Na}^{+}, \mathrm{Li}^{+}$. It is clear that the $\mathrm{ME}-\mathrm{C}^{4} \mathrm{D}$ signal is interfered by a certain degree of noise.

Since the above signal is acquired from the device and the noise is not artificially added, we cannot obtain the original ideal noise-free signal; thus, the evaluation indexes SNR and RMSE cannot be used to quantitatively analyze the denoising effect [31]. For the actual collected $M E-C^{4} \mathrm{D}$ signal, what is really valuable is the peak, especially the peak area or peak height, which reflects the component content of the substance to be measured. Therefore, the peak area or peak height can be used as the evaluation index for denoising effect. Because the peak area can be obtained by multiplying 
TABLE 2: Comparison results of simulation signal denoising with different methods.

\begin{tabular}{lccccc}
\hline Parameter & Savitzky-Golay & Fast Fourier transform & Soft threshold method & Proposed method & SNR of noisy signal (dB) \\
\hline SNR & 54.6092 & 59.2802 & 65.1503 & $\mathbf{7 1 . 4 7 1 5}$ & $\mathbf{0 . 0 1 6 9}$ \\
RMSE & 0.0392 & 0.0310 & 0.0231 & $\mathbf{7 8 . 6 3 9 3}$ & 21 \\
SNR & 64.0419 & 70.9654 & 74.6367 & $\mathbf{0 . 0 1 1 8}$ & 25 \\
RMSE & 0.0245 & 0.0173 & 0.0144 & $\mathbf{8 5 . 7 1 5 3}$ & 29 \\
SNR & 72.1866 & 76.0289 & 0.9725 & $\mathbf{0 . 0 0 8 3}$ & \\
RMSE & 0.0163 & 0.0134 & 0.0105 & \\
\hline
\end{tabular}
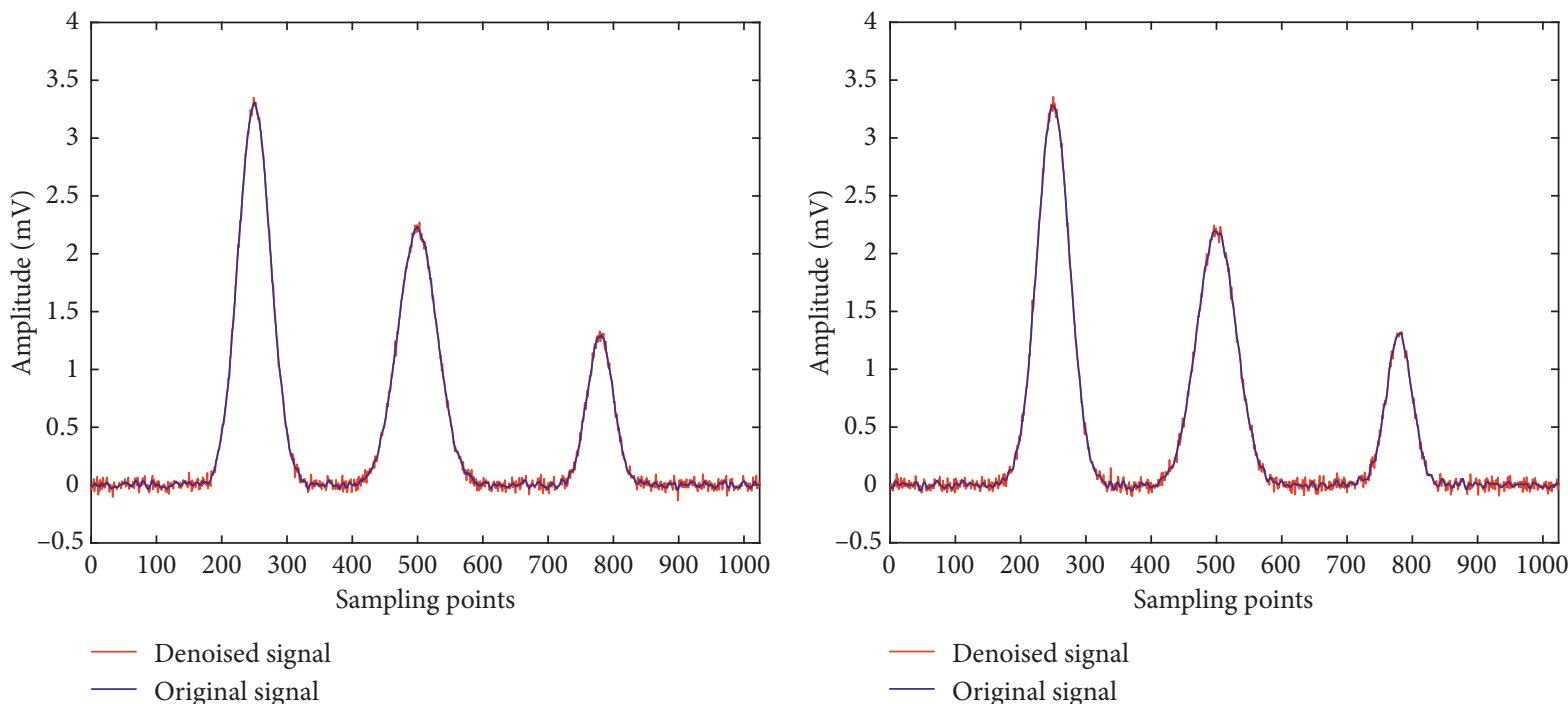

(a)

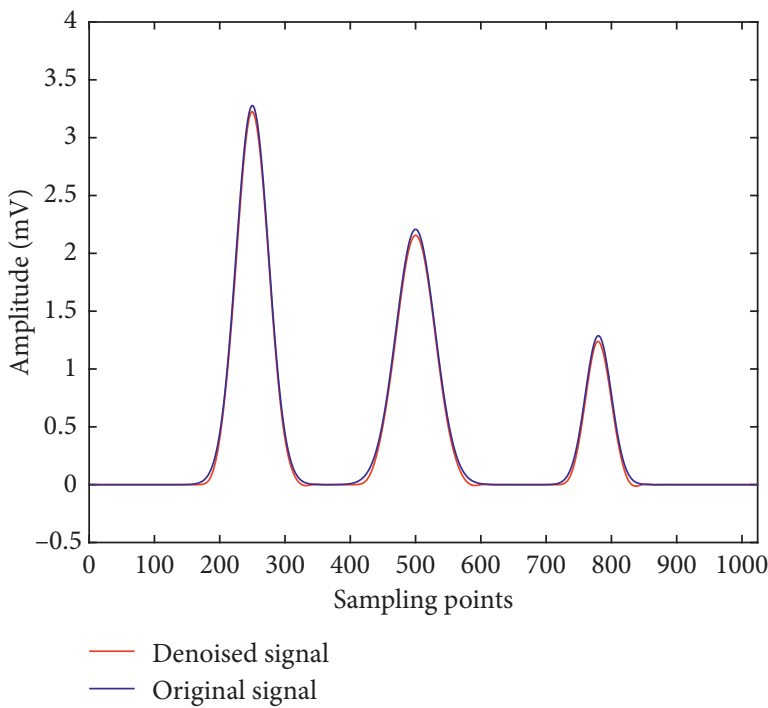

(c)

(b)

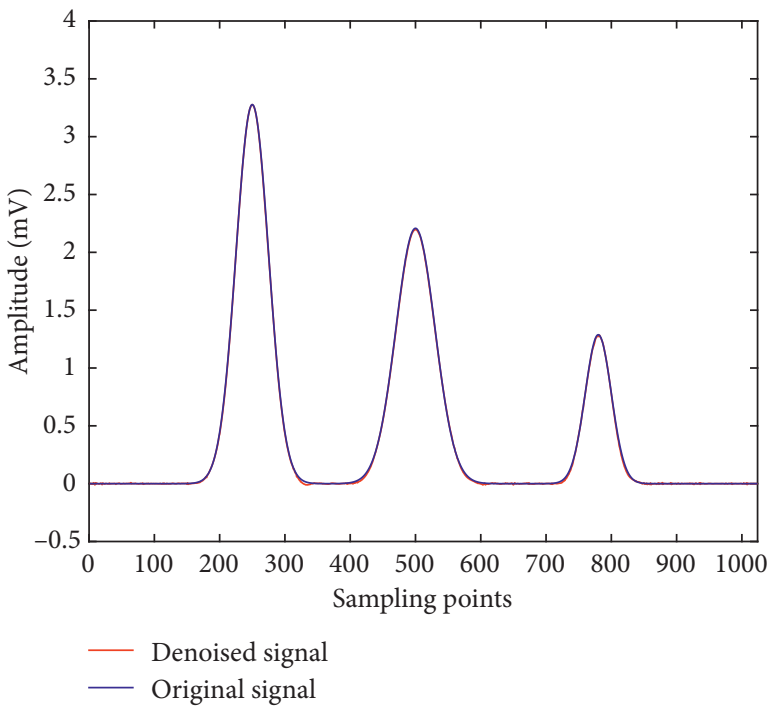

(d)

Figure 8: The results of simulation signal denoising of $29 \mathrm{~dB}$ by different methods. (a) Savitzky-Golay. (b) Fast Fourier Transform. (c) Soft threshold method. (d) The proposed method.

the peak height and the half width, the peak area distortion is used only as the evaluation index to quantitatively analyze the denoising processing effect of the $\mathrm{ME}-\mathrm{C}^{4} \mathrm{D}$ signal. The lower the peak area error is, the lower the distortion is, and the better the denoising effect is.

$$
\Delta A=\left|\frac{\left(A_{d}-A_{o}\right)}{A_{d}}\right| \times 100,
$$

where $A$ is the peak area of ME-C ${ }^{4} D$ signal; subscripts $d$ and $o$ represent before and after signal processing, respectively. 


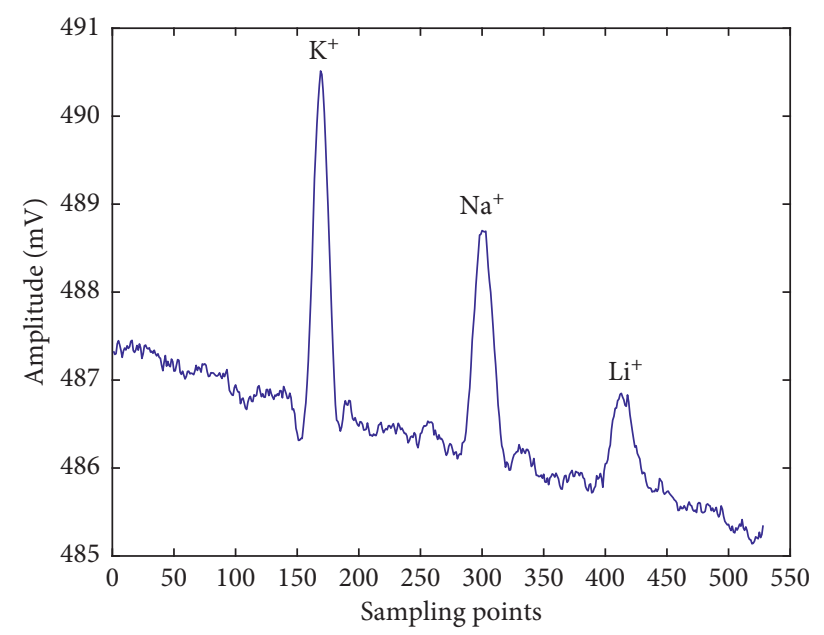

FIGURE 9: Detection signal with concentrations of $0.1 \mathrm{mM}$.

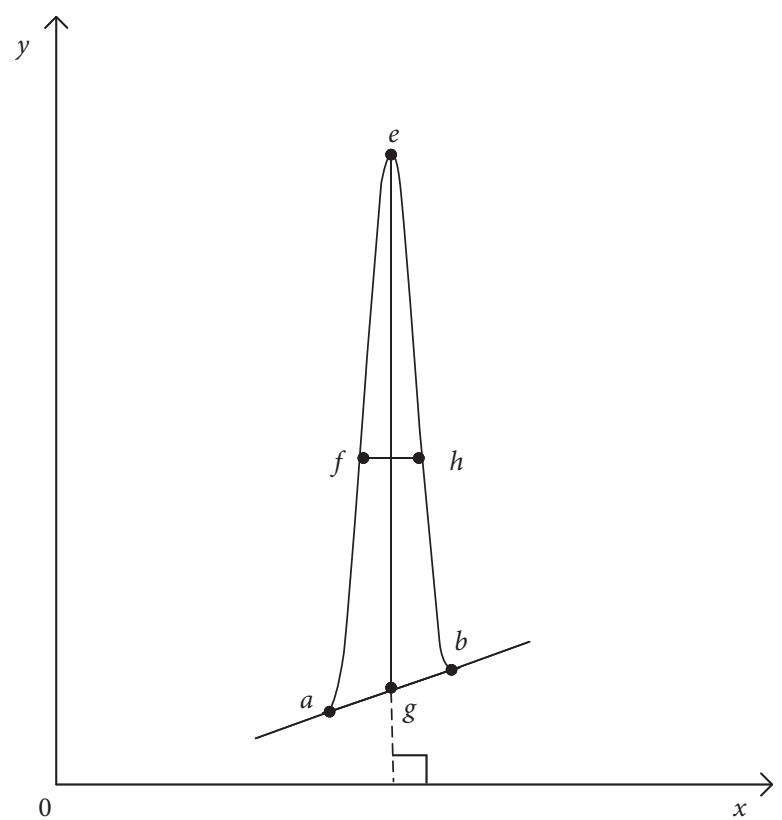

Figure 10: Schematic diagram of peak area calculation method.

Generally speaking, the peak area distortion degree kept within $5 \%$ belongs to the normal range.

For peaks with symmetrical shape and no baseline drift, the peak height is the vertical distance from the peak top to the baseline at the bottom of the peak, and the half peak width is the width at $1 / 2$ the peak height. However, when the peak shape is asymmetrical and there is a baseline drift, the calculation of peak area by the above method will produce a large error. Therefore, this paper uses the following method to calculate the peak area.

The schematic diagram for calculating the peak area is shown in Figure 10. Firstly, the baseline $A B$ is determined by the first derivative method. A threshold value is set, when the first derivative is greater than the threshold, it is determined as the starting point of the peak. When the first derivative changes from positive to negative, it is judged as the apex of the peak. After the apex is determined, if the absolute value of the first derivative value of the signal is less than the threshold, it is defined as the end point of the peak. Then, a line perpendicular to the $x$-axis is drawn from the apex $E$ of the peak. This line intersects the baseline $A B$ at point $G$, and the length of EG is the peak height $\left(h_{E G}\right)$. Finally, a line parallel to the $x$-axis and through the midpoint of the $E G$ is drawn. This line intersects the wave peak at two points, $F$ and $H$, and the length of $F H$ is half width of the peak $\left(\sigma_{F H}\right)$. So, the peak area can be calculated as

$$
A=h_{E G} \times \sigma_{F H} .
$$

The improved threshold function denoising method is adopted to denoise the signal in Figure 9, and the results are shown in Figure 11(d). In order to fully verify the effectiveness of the improved threshold function method for denoising the $\mathrm{ME}-\mathrm{C}^{4} \mathrm{D}$ signal, the denoising results of 


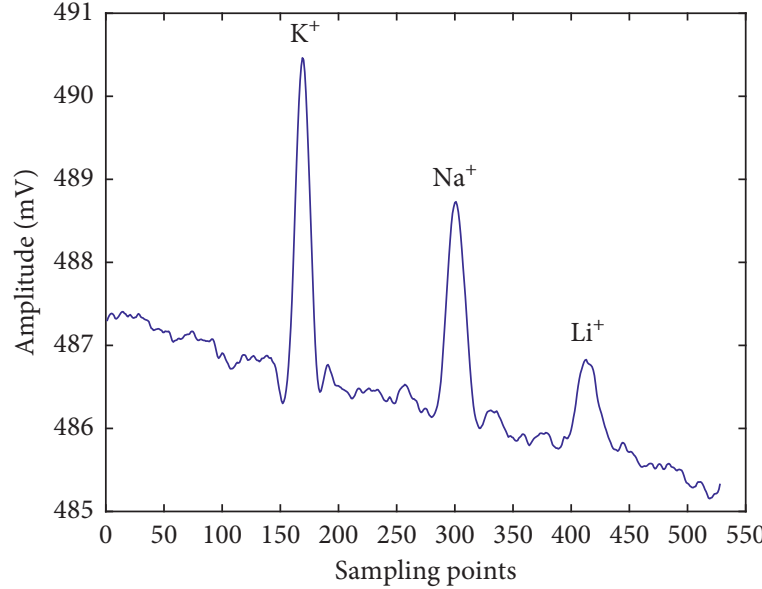

(a)

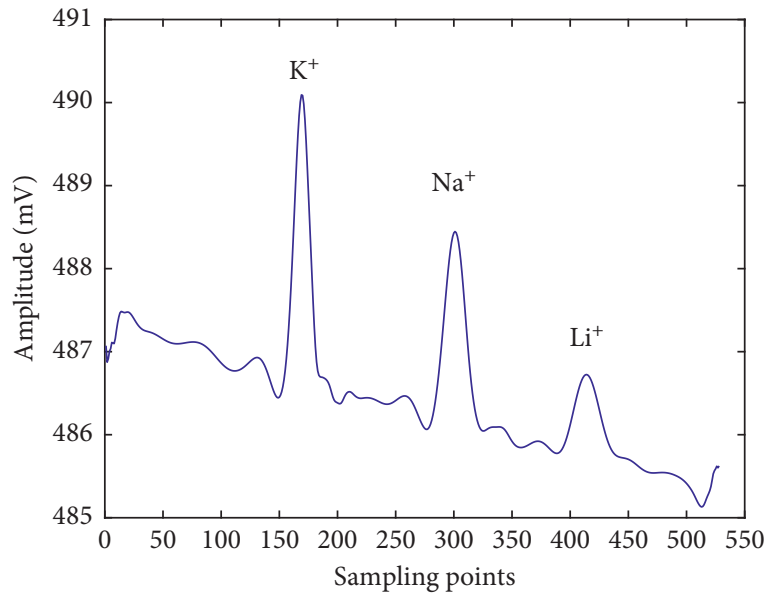

(c)

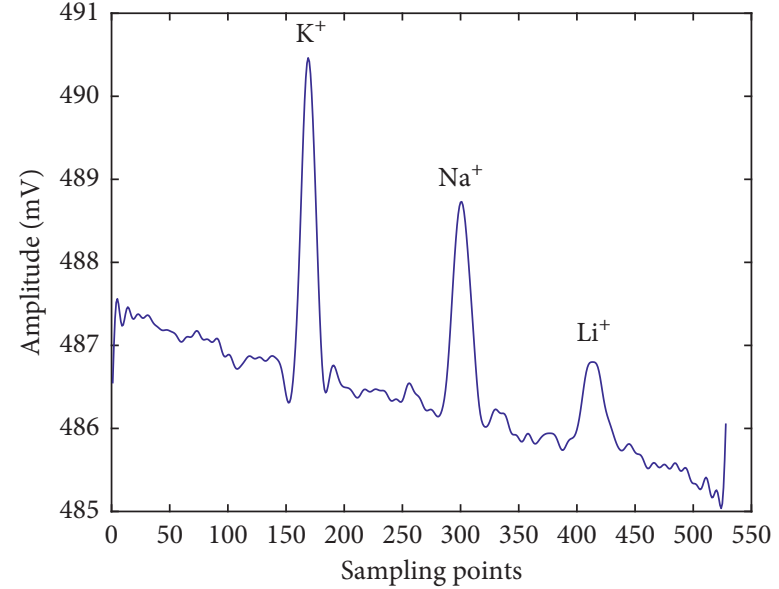

(b)

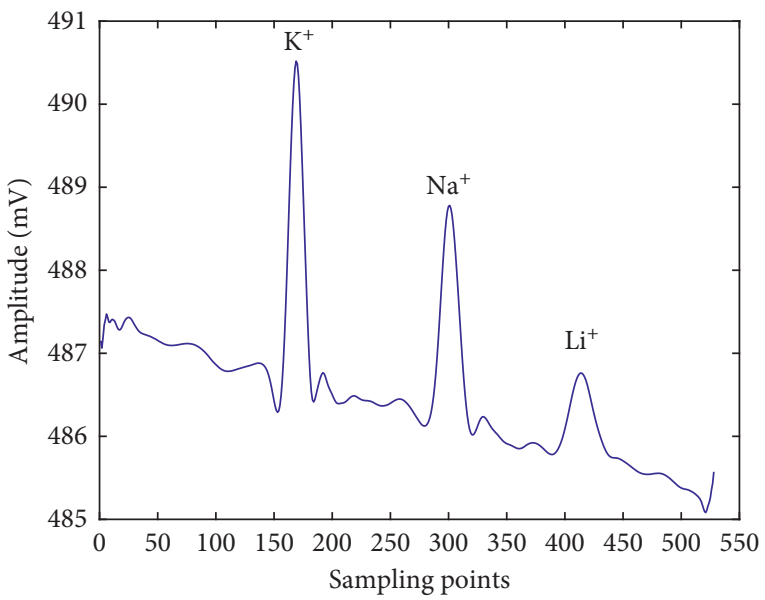

(d)

FIGURE 11: Effect of different methods on signal denoising with concentration of 0.1 mM. (a) Savitzky-Golay. (b) Fast Fourier transform. (c) Soft threshold method. (d) Proposed method.

Savitzky-Golay method, Fast Fourier Transform method, and traditional soft threshold method are used for comparative analysis. The denoising effect is shown in Figures 11(a)-11(c). The peak area distortion degrees after denoising of each method are calculated according to (13) and (14), as shown in Table 3.

It can be seen from the data in Table 3 that after Savitzky-Golay and Fast Fourier Transform are used to denoise the ME- $\mathrm{C}^{4} \mathrm{D}$ signal, the peak area distortion of the third peak of the signal is greater than $5 \%$, and Figures 11(a) and 11(b) are showing that there is still some noise left in the reconstructed signal after denoising, which has a certain impact on the accuracy of the ME- $C^{4} \mathrm{D}$ signal analysis. When using soft threshold function to denoise, the baseline is smooth and the noise is basically removed, but the area distortion of the first peak and the third peak are $10.9102 \%$ and $12.5462 \%$, respectively, which greatly affects the determination of the content of the measured substance. Obviously, the new proposed method can remove the noise well, the denoised signal is smooth, and the peak areas of the three peaks remain basically unchanged. All these above suggest that the
TABLE 3: Peak area distortion after denoising by different methods.

\begin{tabular}{lccc}
\hline Denoising method & \multicolumn{3}{c}{ Peak area distortion } \\
& $\begin{array}{c}\text { First peak } \\
(\%)\end{array}$ & $\begin{array}{c}\text { Second peak } \\
(\%)\end{array}$ & $\begin{array}{c}\text { Third peak } \\
(\%)\end{array}$ \\
\hline $\begin{array}{l}\text { Savitzky-Golay } \\
\begin{array}{l}\text { Fast Fourier } \\
\text { transform }\end{array}\end{array}$ & 0.2532 & 0.7758 & 7.4411 \\
$\begin{array}{l}\text { Soft threshold } \\
\text { method }\end{array}$ & 0.3032 & 0.4154 & 6.2670 \\
Proposed method & 10.9106 & 3.6005 & 12.5462 \\
\hline
\end{tabular}

improved threshold function method in this paper is effective and superior to the existing methods.

\section{Concluding Remarks}

In this work, a method of ME- $\mathrm{C}^{4} \mathrm{D}$ signal denoising based on an improved threshold function of wavelet transform is proposed. The simulation experiment results suggest that the proposed method in this paper is superior to Savitzky-Golay, 
Fast Fourier Transform, and soft threshold method. The study on the denoising of the actual detection signals of samples with concentrations of $0.1 \mathrm{mM}$ proves that the proposed method has excellent denoising effect and powerful peak area preservation ability. Therefore, this method has important practical value for signal denoising of $\mathrm{ME}-\mathrm{C}^{4} \mathrm{D}$. Furthermore, the improved threshold function method has some limitations, such as the value of the variable parameter $a$ is manually selected by our experience. It is expected that an algorithm will be designed in the future to realize parameter optimization.

\section{Abbreviation}

ME- Microchip electrophoresis based on capacitively $\mathrm{C}^{4} \mathrm{D}$ : coupled contactless conductivity detection.

\section{Data Availability}

The data used to support the findings of this study are available from the corresponding author upon request.

\section{Conflicts of Interest}

The authors declare that they have no conflicts of interest regarding the publication of this paper.

\section{Acknowledgments}

This work was supported by the Science and Technology Program of Hunan Province (nos. 2017SK2164 and 2019TP1014), the Research Project of Education Department of Hunan Province for Excellent Youth (no. 16B114), the Hunan Provincial Innovation Foundation for Postgraduate (no. CX20190931), and the Scientific Research Innovation Team of Hunan Institute of Science and Technology (no. 2019-TD-10).

\section{References}

[1] K. A. Mahabadi, I. Rodriguez, C. Y. Lim et al., "Capacitively coupled contactless conductivity detection with dual top-bottom cell configuration for microchip electrophoresis," Electrophoresis, vol. 31, no. 6, pp. 1063-1070, 2010.

[2] K. C. A. Rezende, R. C. Moreira, L. P. L. Logrado et al., "Authenticity screening of seized whiskey samples using electrophoresis microchips coupled with contactless conductivity detection," Electrophoresis, vol. 37, no. 21, pp. 2891-2895, 2016.

[3] F. Q. Nie, M. Macka, and B. Paull, "Micro-flow injection analysis system: on-chip sample preconcentration, injection and delivery using coupled monolithic electroosmotic pumps," Lab on A Chip, vol. 7, no. 11, 2007.

[4] A. Manz, N. Graber, and H. M. Widmer, "Miniaturized total chemical analysis systems: a novel concept for chemical sensing," Sensors and Actuators B (Chemical), vol. 1, no. 1-6, pp. 244-248, 1990.

[5] T. Vilkner, D. Janasek, and A. Manz, "Micro total analysis systems. Recent developments," Analytical Chemistry, vol. 76, no. 12, pp. 3373-3386, 2004.

[6] G. F. Christopher and S. L. Anna, "Topical review: microfluidic methods for generating continuous droplet streams,"
Journal of Physics D Applied Physics, vol. 40, no. 19, pp. 3373-3386, 2007.

[7] D. Liu, H. Zhang, F. Fontana, J. T. Hirvonen, and H. A. Santos, "Current developments and applications of microfluidic technology toward clinical translation of nanomedicines," Advanced Drug Delivery Reviews, vol. 128, pp. 54-83, 2018.

[8] H. Kimura, Y. Sakai, and T. Fujii, "Organ/body-on-a-chip based on microfluidic technology for drug discovery," Drug Metabolism and Pharmacokinetics, vol. 33, no. 1, pp. 43-48, 2017.

[9] H. Yu, G. Z. Cai, L. Y. Zheng et al., "A microfluidic signal-off biosensor for rapid and sensitive detection of Salmonella using magnetic separation and enzymatic catalysis," Food Control, vol. 103, pp. 186-193, 2019.

[10] G. Chen, Y. Lin, and J. Wang, "Microchip capillary electrophoresis with electrochemical detection for monitoring environmental pollutants," Current Analytical Chemistry, vol. 2, no. 1, pp. 43-50, 2006.

[11] L. Mou, R. H. Dong, B. F. Hu et al., "Hierarchically structured microchip for point-of-care immunoassays with dynamic detection ranges," Lab on a Chip, vol. 19, 2019.

[12] R. M. Guijt, E. Baltussen, G. van der Steen et al., "Capillary electrophoresis with on-chip four-electrode capacitively coupled conductivity detection for application in bioanalysis," Electrophoresis, vol. 22, no. 12, pp. 2537-2541, 2001.

[13] K. Pavel and P. C. Hauser, "Capacitively coupled contactless conductivity detection for microseparation techniques-recent developments," Electrophoresis, vol. 32, no. 1, pp. 30-42, 2011.

[14] W. K. T. Coltro, R. S. Lima, T. P. Segato et al., "Capacitively coupled contactless conductivity detection on microfluidic systems-ten years of development," Analytical Methods, vol. 4, no. 1, 2012.

[15] Z. Huang, M. Yang, H. You, and Y. Xie, "Concurrent determination and separation of inorganic cations and anions in microchip electrophoresis with precisely controlled highvoltage," Electrophoresis, vol. 39, no. 14, pp. 1802-1807, 2018.

[16] K. Pavel and P. C. Hauser, "A review of the recent achievements in capacitively coupled contactless conductivity detection," Analytica Chimica Acta, vol. 607, no. 1, pp. 15-29, 2008.

[17] F. Georg, H. Anna, S. Walter et al., "End-to-end differential contactless conductivity sensor for microchip capillary electrophoresis," Analytical Chemistry, vol. 82, no. 8, pp. 32703275, 2010.

[18] W. Cao, X. Chen, X. Yang, and E. Wang, "Discrete wavelets transform for signal denoising in capillary electrophoresis with electrochemiluminescence detection," Electrophoresis, vol. 24, no. 18, pp. 3124-3130, 2003.

[19] B.-F. Liu, Y. Sera, N. Matsubara, K. Otsuka, and S. Terabe, "Signal denoising and baseline correction by discrete wavelet transform for microchip capillary electrophoresis," Electrophoresis, vol. 24, no. 18, pp. 3260-3265, 2003.

[20] Y. N. Tong, Y. G. He, H. M. Li et al., "Method for wavelet transformation using switched-current circuit with approximation optimization in frequency domain," Journal of Electronic Measurement and Instrument, vol. 28, no. 3, pp. 262-271, 2014.

[21] C. Gao, M. Shen, X. Liu, L. Wang, and M. Chu, "End-point static control of basic oxygen furnace (BOF) steelmaking based on wavelet transform weighted twin support vector regression," Complexity, vol. 2019, Article ID 7408725, 16 pages, 2019.

[22] H. F. Zhang, X. W. Liu, X. H. Shao et al., "The study of the improved wavelet thresholding with translation invariant de- 
noising on capillary electrophoresis signal," in Proceedings of the 2009 4th IEEE International Conference on Nano/Micro Engineered and Molecular Systems, Shenzhen, China, 2009.

[23] X. Y. Chen and J. Y. Mo, "Application of lifting-scheme based wavelet transform to the chemical signals," Journal of Instrumental Analysis, vol. 23, no. 1, pp. 5-8, 2004.

[24] C. Chen, W. Teng, and J. H. Hahn, "Nanoband electrode for high-performance in-channel amperometric detection in dual-channel microchip capillary electrophoresis," Electrophoresis, vol. 32, no. 8, pp. 838-843, 2011.

[25] J. Y. Chen, Y. W. Zhan, H. Y. Cao et al., "Wavelet denoising based on continuous differentiable threshold function and scale threshold," Journal of Electronic Measurement and Instrument, vol. 32, no. 10, pp. 169-176, 2018.

[26] G. W. Chen, X. B. Liu, D. Wang et al., "Denoising of MEMS gyroscope based on improved wavelet transform," Journal of Electronics \& Information Technology, vol. 41, no. 5, pp. 1025-1031, 2019.

[27] J. W. Zhang, Y. Feng, and W. Li, "Research on wavelet denoising method based on an improved threshold function," Electronic Design Engineering, vol. 25, no. 9, pp. 137-140, 2017.

[28] W. Wu, "Research and implementation of capillary electrophoresis signal acquisition and storage system based on uTAS," Master thesis, Shanghai University, Shanghai, China, 2006.

[29] W. P. Yan, C. C. Xu, and J. H. Li, "The use of discrete wavelets for signal denoising in microchip capillary electrophoresis," in Proceedings of the 2006 6th World Congress on Intelligent Control and Automation, vol. 7, pp. 5254-5258, Dalian, China, 2006.

[30] H. Xing and Y. Yan, "Detection of low-flying target under the sea clutter background based on volterra filter," Complexity, vol. 2018, Article ID 1513591, 12 pages, 2018.

[31] H. Y. Li, Y. L. Zhou, F. Tian et al., "Wavelet-based vibration signal denoising algorithm with a new adaptive threshold function," Chinese Journal of Scientific Instrument, vol. 36, no. 10, pp. 2200-2206, 2015. 